\title{
Gamma Knife Radiosurgery Using Co-Registration with PET-CT and MRI for Recurrent Nasopharyngeal Carcino- ma with Previous Radiotherapy : A Single Center 14-Year Experience
}

\author{
Chaejin Lee, ${ }^{1,2}$ Seong-Hyun Park, ${ }^{1,2}$ Sang-Youl Yoon, ${ }^{1,2}$ Ki-Su Park, ${ }^{1,2}$ Jeong-Hyun Hwang, ${ }^{1,2}$ Sung-Kyoo Hwang ${ }^{1,2}$ \\ Department of Neurosurgery,' School of Medicine, Kyungpook National University, Daegu, Korea \\ Department of Neurosurgery, Kyungpook National University Hospital, Daegu, Korea
}

Objective : We retrospectively evaluated the efficacy of Gamma Knife radiosurgery (GKS) for recurrent nasopharyngeal carcinoma (NPC) in patients who previously underwent radiotherapy, and analyzed the treatment outcomes over 14 years.

Methods : Ten patients with recurrent NPC who had previously received radiotherapy underwent stereotactic radiosurgery using a Gamma Knife ${ }^{\oplus}$ (Elekta Inc, Atlanta, GA, USA) between 2005 and 2018. The median target volume was $8.2 \mathrm{~cm}^{3}$ (range, 1.7-17.8), and the median radiation dose to the target was $18 \mathrm{~Gy}$ (range, 12-30). The median follow-up period was 18 months (range, 6-76 months). Overall and local failure-free survival rates were determined using the Kaplan-Meier method.

Results : The NPCs recurred at the primary cancer site in seven patients (70\%), as distant brain metastasis in two (20\%), and as an extension into brain in one (10\%). The recurrent tumors in seven of the 10 patients $(70 \%)$ were found on the routine follow-up imaging studies. Two patients presented with headache and one with facial pain. Local failure after GKS occurred in five patients (50\%) : two of whom died eight and 6 months after GKS, respectively. No adverse radiation effects were noted after GKS. The 1- and 3-year overall survival rates after GKS were $90 \%$ and $77 \%$, respectively. The local failure-free survival rates at 6 months, 1 year, and 3 years after GKS were $80 \%, 48 \%$, and 32\%, respectively. The median interval from GKS to local failure was 8 months (range, 6-12). Univariate analysis revealed that using co-registration with positron emission tomography-computed tomography (PET-CT) and magnetic resonance imaging (MRI) was associated with a lower local failure rate of recurrent NPC $(p=0.027)$.

Conclusion : GKS is an acceptable salvage treatment option for patients with recurrent NPC who previously received radiation therapy. PET-CT and MRI co-registration for dose planning can help achieve local control of recurrent NPC.

Key Words : Radiosurgery · Nasopharyngeal carcinoma · Positron emission tomography computed tomography.

- Received : January 5, 2019 •Revised : April 24, $2019 \bullet$ Accepted : June 4, 2019

- Address for reprints : Seong-Hyun Park

Department of Neurosurgery, School of Medicine, Kyungpook National University, Department of Neurosurgery, Kyungpook National University Hospital, 130 Dongdeok-ro, Jung-gu, Daegu 41944, Korea

Tel : +82-53-200-5652, Fax : +82-53-423-0504, E-mail : nsdoctor@naver.com, ORCID : https://orcid.org/0000-0002-0982-5338

This is an Open Access article distributed under the terms of the Creative Commons Attribution Non-Commercial License (http://creativecommons.org/licenses/by-nc/4.0) which permits unrestricted non-commercial use, distribution, and reproduction in any medium, provided the original work is properly cited. 


\section{INTRODUCTION}

Nasopharyngeal carcinoma (NPC) is a common head and neck malignancy in East Asian countries, especially in China. Early-stage NPC is curable, and external beam radiotherapy (EBRT) alone is sufficient treatment given the tumor's radiosensitivity. However, late-stage NPC remains a challenge because of its high rate of recurrence. Late-stage NPC has conventionally been treated with concurrent chemoradiotherapy (CCRT). Many treatment options for recurrent NPCs have been introduced to improve local control, including fractionated stereotactic radiotherapy (FSRT), intensity-modulated radiosurgery (IMRT), brachytherapy, and stereotactic radiosurgery (SRS) ${ }^{1,3-6,8,9,11-14)}$. SRS using Gamma Knife ${ }^{\circledR}$ (Elekta Inc, Atlanta, GA, USA) has become an alternative treatment for the majority of recurrent or residual brain tumors, particularly those that underwent previous conventional radiotherapy that renders additional irradiation difficult. Gamma Knife radiosurgery (GKS) has the advantage of high conformity to intracranial tumors and the sparing of surrounding normal tissue from irradiation, thereby reducing radiation-related complications. However, GKS is often inadequate for achieving local control in patients with recurrent NPC because of inaccurate target delineation, and has rarely been used for recurrent $\mathrm{NPC}^{6,10)}$. To improve local control in patients with re- current NPC using GKS, we employed co-registration with positron emission tomography-computed tomography (PET$\mathrm{CT}$ ) and magnetic resonance imaging (MRI). PET-CT is valuable for differentiating recurrent NPC from non-tumorous tissues because of its specific uptake in the tumor ${ }^{7,10)}$. To the best of our knowledge, there have been no studies of PET-CT and MRI co registration when using GKS to improve local control of recurrent NPC. In this study, we evaluated treatment outcomes following GKS using co-registration with PET-CT and MRI in patients with recurrent NPC, and investigated factors that are associated with local tumor control.

\section{MATERIALS AND METHODS}

\section{Study design}

We conducted a single-institution, retrospective study that was approved by the Institutional Review Board of Kyungpook National University Hospital (KNUH 2014-03-013-001). The overall outcomes of 10 consecutive patients with recurrent NPC who underwent SRS with the Leksell Gamma Knife $^{\circledast}$ (Elekta Inc) at our hospital between 2005 and 2018 were evaluated. The diagnosis of initial and recurrent NPC was confirmed by biopsy and imaging studies by head-neck surgeons. All patients were staged according to the 2002
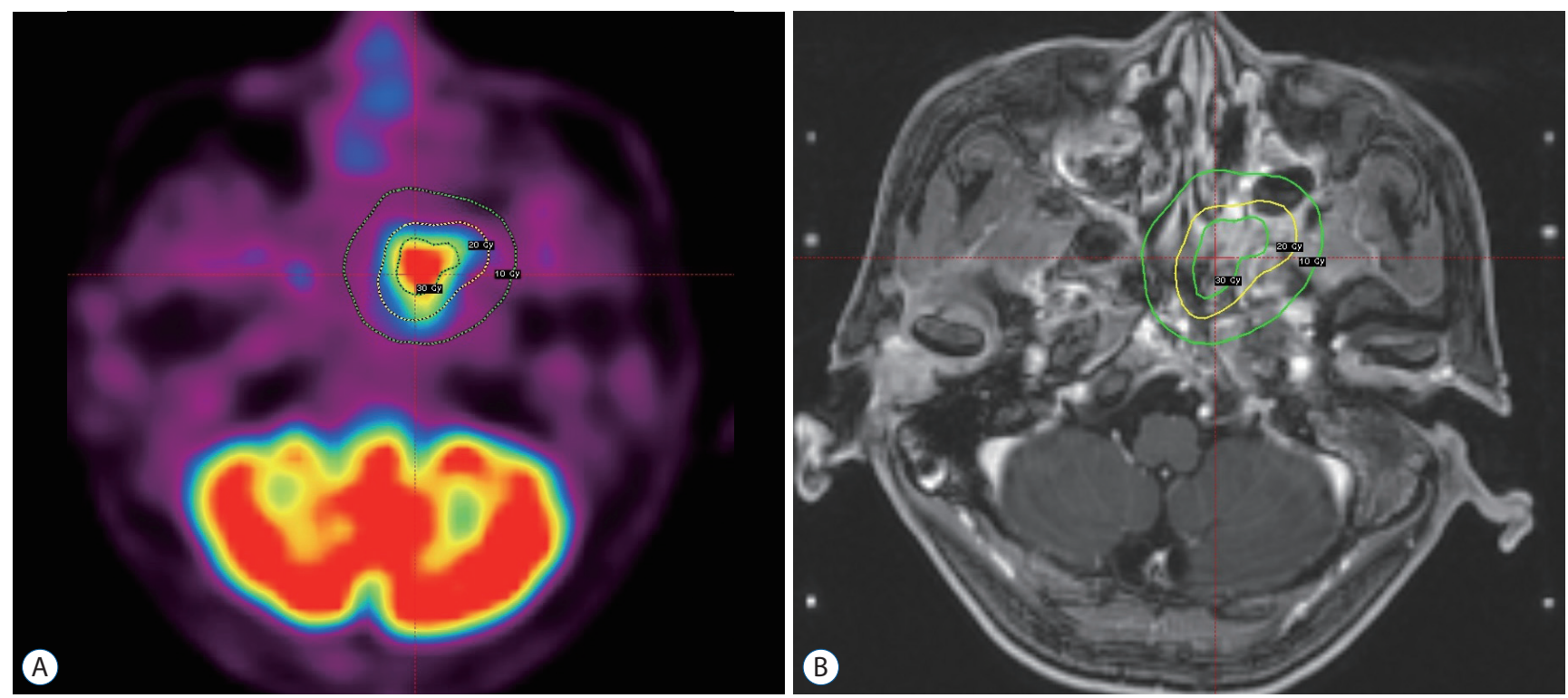

Fig. 1. A : Dose planning for recurrent nasopharyngeal carcinoma showing co-registration with PET-CT and MRI. The tumor was treated with a radiation dose of 20 Gy at the 50\% isodose line. B : Dose planning for recurrent nasopharyngeal carcinoma showing MRI without co-registration with PET-CT. PET-CT : positron emission tomography-computed tomography, MRI : magnetic resonance imaging. 
Union for International Cancer Control (UICC) staging system, and all underwent conventional CCRT. ${ }^{18} \mathrm{~F}$-fluoro-2-deoxy-D-glucose PET-CT was performed for all patients to assess the recurrence status of NPC, as well as its extent (including distant metastasis). GKS was recommended when radiation-related risks existed after conventional radiotherapy or when patients refused other salvage treatments such as surgery, chemotherapy, and conformal radiotherapy. Recurrent NPCs that were suitable for analysis were identified using our GKS database and were verified on imaging studies conducted by a neuroradiologist. Outcome data were collected through an independent review of the patients' medical records and were analyzed by neurosurgeons who did not participate in their management.

\section{GKS using the PET-CT and MRI co-registration technique}

A Model G Leksell stereotactic frame was applied to all patients under local anesthesia. After attaching an imagingcompatible fiducial system to the frame, we performed high resolution stereotactic MRI for all patients. We were unable to obtain stereotactic PET-CT images at our hospital because of the lack of frame-based PET-CT facilities. Instead, we performed high resolution PET-CT without the frame. Images were transferred to a computer workstation for treatment planning; stereotactic MR images were fused with PET-CT images using the co-registration technique. Recurrent NPC was outlined using optimized imaging integration of stereotactic MRI and conventional PET-CT (Fig. 1). Six patients $(60 \%)$ underwent surgery and CCRT following their initial diagnosis at our hospital, while four (40\%) were referred from other hospitals at the time of NPC recurrence; the former six patients underwent GKS using co-registration with PET-CT and MRI, while the latter four did not undergo GKS using this technique because their PET-CT images obtained at other hospitals were not adjustable for co-registration on our hospital's workstation computer. It was difficult to accurately delineate the tumor volume on MRI without co-registration with PET-CT (Fig. 2).

The target volume was determined at the time of treatment planning with the goal of enveloping the 3-dimensional tumor volume in a highly conformed and selective dose plan. All patients received an intravenous dose of 20-40 mg methyl prednisolone after GKS, and all were discharged from the hospital within 6-24 hours.

\section{Follow-up examination}

The patients were instructed to undergo clinical follow-up examinations every 1-3 months after GKS as well as an imaging assessment (MRI and CT) every 6 months; neurological and nasopharyngeal examinations were performed at the time of imaging to assess clinical outcomes. Local failure was de-
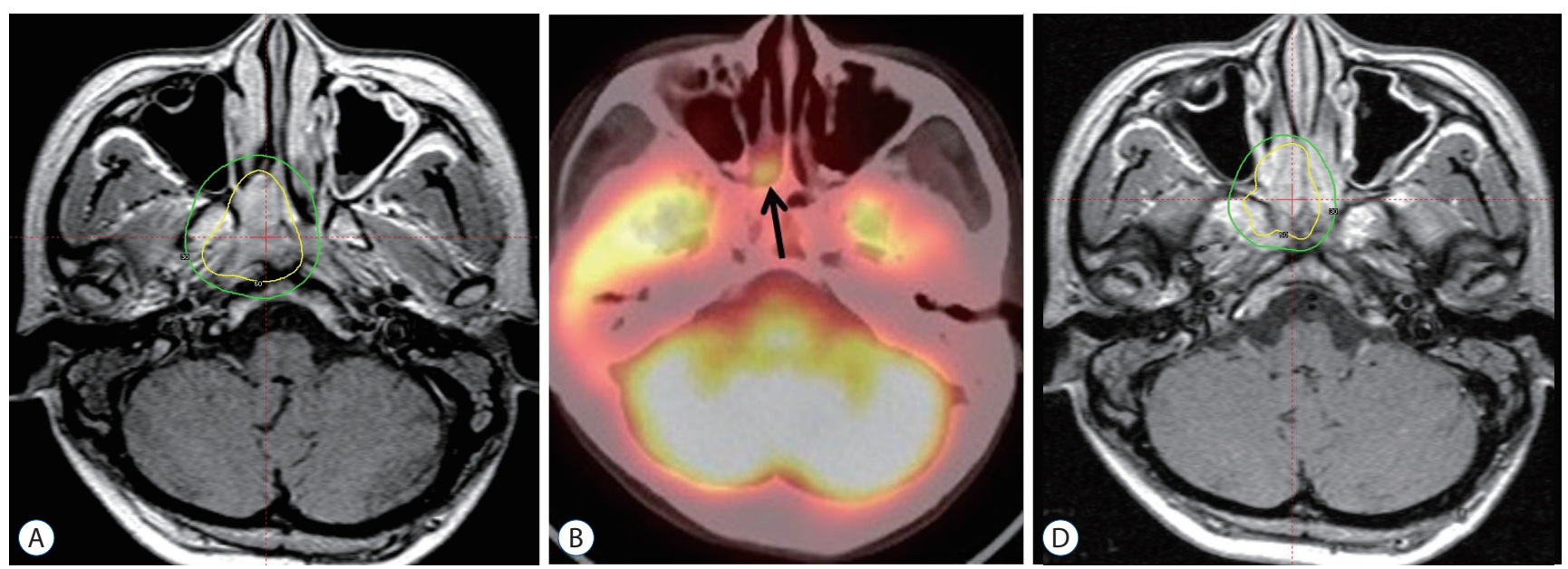

Fig. 2. A : Dose planning for recurrent nasopharyngeal carcinoma showing MRI without co-registration with PET-CT. It was difficult to accurately define the tumor on MRI without co-registration with PET-CT. PET-CT images obtained at other hospitals were not adjustable for co-registration on our hospital's workstation computer. The ill-defined tumor was treated with a radiation dose of $18 \mathrm{~Gy}$ at the $50 \%$ isodose line. B : PET-CT showing tumor recurrence (arrow) 8 months after Gamma Knife radiosurgery. C : The tumor was re-treated with a radiation dose of $20 \mathrm{~Gy}$ at the $50 \%$ isodose line. MRI : magnetic resonance imaging, PET-CT: positron emission tomography-computed tomography. 
fined based on serial MR or CT with increased contrast enhancement and radiographic progression. All patients received a minimum of 6 months of follow-up (median, 18 months; range, 6-76 months). Five patients (50\%) underwent followup for longer than 2 years.

\section{Statistical analysis}

We constructed Kaplan-Meier plots for overall survival and local failure-free survival rates calculated from the date of GKS until that of the last follow-up examinations. The following variables were assessed : age, sex, clinical presentation, tumor staging, lesion location and volume, duration until recurrence, margin dose, maximal dose, isodose line, number of isocenters, conformity index and co-registration technique with PET-CT. A probability value less than 0.05 was considered significant. Univariate analysis was performed using the log-rank test for categorical data and Cox proportional hazards model for continuous data. Variables were compared using Fisher's exact test and the Mann-Whitney test. The SPSS software version 20.0 (IBM Corp, Armonk, NY, USA) was used for all analyses.

\section{RESULTS}

\section{Patients}

Patient characteristics are summarized in Tables 1 and 2. All patients (eight men and two women) had histologically confirmed NPC; their median age was 56 years (range, 34-73 years). All patients had undergone CCRT on initial NPCs diagnosis. None previously received brachytherapy. Cisplatin-based chemotherapy was administered to all patients; however, the regimen details differed among hospitals and physicians. The median duration between initial NPC diagnosis and recurrence was 20 months (range, 5-161). The most common locations of recurrence were the nasopharyngeal region. Seven NPCs (70\%) were locally recurrent tumors at the same original site, two $(20 \%)$ were distant metastases on the cavernous sinus and cerebellum, and one (10\%) extended into the frontal lobe. The patient with distant metastasis in the cerebellum had undergone a craniotomy for a brain tumor that was histologically confirmed as metastasis from NPC. Recurrence was diagnosed during routine follow-up imaging in seven patients (four via PET-CT, two via MRI, and one via CT). Of the remaining three patients, two presented with a headache and lhad facial pain at recurrence. According to the UICC staging system, four patients (40\%) had early-stage (I-II) disease and six had late-stage (III-IV).

The median target volume was $8.2 \mathrm{~cm}^{3}$ (range, 1.7-17.8), and the median prescription dose delivered to the tumor margin was 18 Gy (range, 12-30). The prescribed isodose line for all patients was $50 \%$, and the maximum radiation dose ranged from 24 to $60 \mathrm{~Gy}$ (median, 36). One patient with a large tumor underwent hypofractionated GKS (three fractions) and the remaining nine underwent single-session GKS.

\section{Clinical outcomes}

Five patients (50\%) experienced local failure after GKS

Table 1. Summary of patient, recurrent nasopharyngeal carcinoma, and radiosurgery characteristics

\begin{tabular}{lc}
\hline Variable & Value \\
\hline Age (years) & $56(34-73)$ \\
Gender (male : female) & $8: 2$ \\
Previous concurrent chemo-radiation & $10(100)$ \\
Finding at recurrence & \\
Follow-up imaging study & $7(70)$ \\
$\quad$ PET-CT & $4(40)$ \\
$\quad$ MRI & $2(20)$ \\
$\quad$ CT & $1(10)$ \\
Headache & $2(20)$ \\
Facial pain & $1(10)$ \\
Duration (months) & \\
From initial diagnosis to recurrence & $20(5-161)$ \\
From radiosurgery to recurrence & $8(6-12)$ \\
Stage (UICC staging system) & \\
Early (I-II) & 4 \\
Late (III-IV) & 6.2 \\
Co-registration with PET-CT & 18 \\
Tumor volume, median (cm ${ }^{3}$ ) & 36 \\
Margin dose, median (Gy) & 50 \\
Maximal dose, median (Gy) & 90 sodose line, median (\%) \\
\hline Number of isocenters, median & \\
\hline & \\
\hline
\end{tabular}

Values are presented as median (range) or number (\%) unless otherwise indicated. PET-CT : positron emission tomography-computed tomography, MRI : magnetic resonance imaging, UICC : Union for International Cancer Control 
while the remaining five (50\%) had not experienced such failure as of their final follow-up visit. Of the five patients who experienced local failure after GKS, three underwent additional GKS once, twice, and thrice, respectively; moreover, the fourth underwent additional GKS and endoscopic surgery while the fifth was treated with conservative therapy. Two of the five patients with local failure underwent GKS using coregistration with PET-CT while four of the five patients without local failure underwent GKS using the co-registration technique. Furthermore, two of five patients who experienced local failure died eight and 6 months after GKS, respectively, one of local failure while the other of an unspecified cause. There were no symptomatic radiation-related complications from salvage GKS. The overall survival rates post-GKS were $90 \%$ at 1 year and $77 \%$ at 3 years, while the local failure-free survival rates post-GKS were $80 \%, 48 \%$, and $32 \%$ at 6 months, 1 year, and 3 years, respectively (Fig. 3). The median interval between GKS and tumor recurrence was 8 months (range, 6-12). There were no significant differences in the local failure rate according to age, sex, clinical presentation, tumor staging, lesion location and volume, duration until recurrence, margin dose, maximal dose, number of isocenters, and conformity index. On univariate analysis, patients who underwent GKS using co-registration with PET-CT and MRI had significantly better tumor control rates than those who underwent the procedure using conventional GKS planning $(p=0.027)$. Kaplan-Meier analysis showed that GKS using coregistration with $\mathrm{PET}-\mathrm{CT}$ was associated with better tumor control in terms of NPC recurrence (Fig. 4).

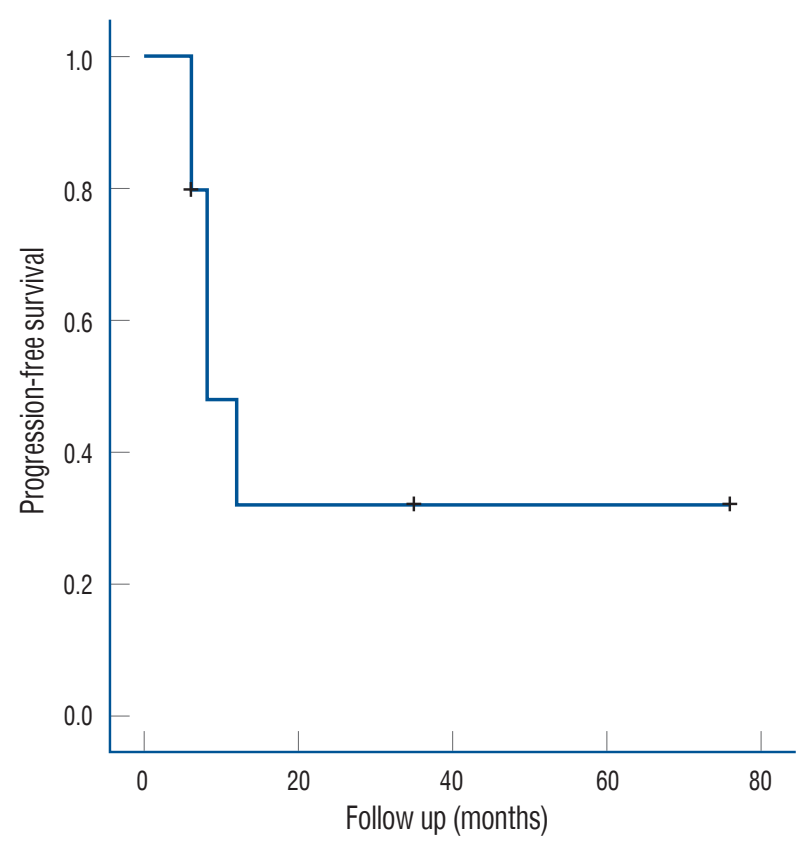

Fig. 3. Kaplan-Meier curve showing local failure-free survival of patients with recurrent nasopharyngeal carcinoma after Gamma Knife radiosurgery.

Table 2. Individual data of patients with recurrent nasopharyngeal carcinoma treated with GKS

\begin{tabular}{|c|c|c|c|c|c|c|c|c|c|c|c|}
\hline $\begin{array}{c}\text { Patient } \\
\text { No. }\end{array}$ & Sex & $\begin{array}{c}\text { Age } \\
\text { (years) }\end{array}$ & Stage & $\begin{array}{l}\text { Treatment } \\
\text { after initial } \\
\text { operation }\end{array}$ & Recurred site & $\begin{array}{c}\text { Symptoms } \\
\text { at } \\
\text { recurrence }\end{array}$ & $\begin{array}{l}\text { Co- } \\
\text { registration } \\
\text { with } \mathrm{PET}\end{array}$ & $\begin{array}{c}\text { Tumor } \\
\text { volume } \\
\left(\mathrm{cm}^{3}\right)\end{array}$ & $\begin{array}{l}\text { Margin } \\
\text { dose (Gy) }\end{array}$ & $\begin{array}{l}\text { Time to } \\
\text { recur } \\
\text { after GKS } \\
\text { (months) }\end{array}$ & $\begin{array}{c}\text { Treatment } \\
\text { after } \\
\text { recurrence }\end{array}$ \\
\hline 1 & $\mathrm{~F}$ & 49 & Early & CCRT & Frontal lobe & Headache & No & 7.8 & 12 & 6 & Conservative \\
\hline 2 & M & 73 & Early & CCRT & Local & Headache & Yes & 8.8 & 15 & - & \\
\hline 3 & M & 44 & Late & CCRT & Cerebellum & No & No & 17.8 & $\begin{array}{c}30 \text { in } \\
3 \text { fraction }\end{array}$ & - & \\
\hline 4 & $\mathrm{~F}$ & 39 & Late & CCRT & Local & No & No & 9.9 & 18 & 8 & GKS+surgery \\
\hline 5 & M & 56 & Early & CCRT & Local & No & Yes & 13.2 & 20 & - & \\
\hline 6 & $M$ & 60 & Late & CCRT & $\begin{array}{l}\text { Cavernous } \\
\text { sinus }\end{array}$ & No & Yes & 5.5 & 16 & - & \\
\hline 7 & M & 66 & Early & CCRT & Local & No & Yes & 8.1 & 16 & 12 & GKS \\
\hline 8 & M & 34 & Late & CCRT & Local & Facial pain & No & 1.7 & 18 & 6 & GKS \\
\hline 9 & M & 66 & Late & CCRT & Local & No & Yes & 1.8 & 20 & 8 & GKS \\
\hline 10 & $M$ & 55 & Late & CCRT & Local & No & Yes & 8.3 & 20 & - & \\
\hline
\end{tabular}

GKS : Gamma Knife radiosurgery, PET : positron emission tomography, F : female, CCRT : concurrent chemoradiotherapy, M : male 


\section{DISCUSSION}

Overall survival rates in patients with NPC are poor even with the availability of modern advanced technologies; the majority of the patients experience tumor recurrence that leads to morbidity and mortality ${ }^{11)}$. In this study, we reviewed the clinical and radiological outcomes of patients who underwent salvage GKS for recurrent NPC and analyzed the factors that were associated with local failure post-GKS. We found that GKS for recurrent NPC produced effective local control

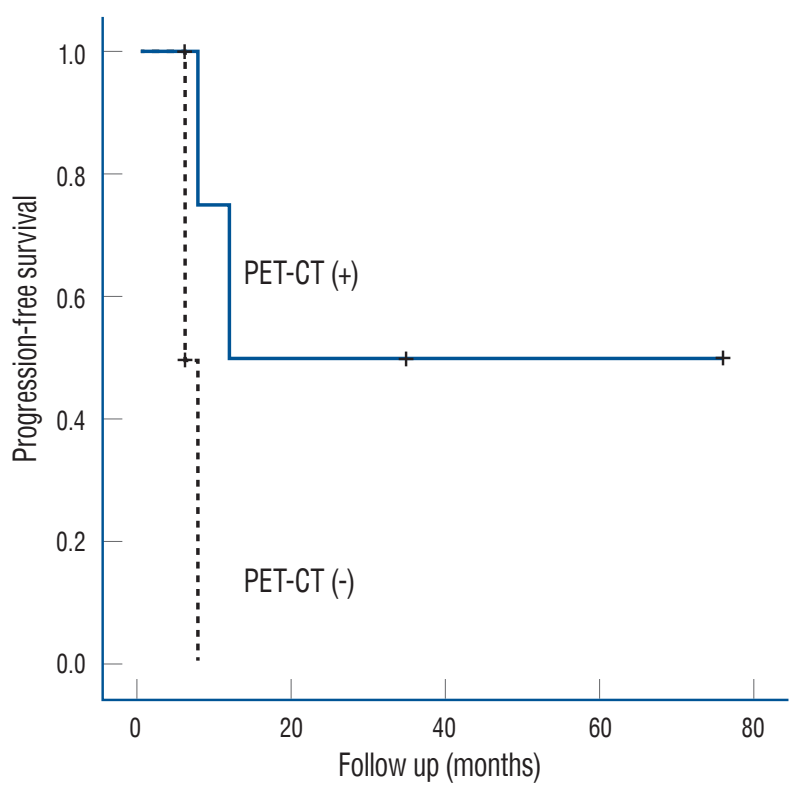

Fig. 4. Kaplan-Meier curves showing local failure-free survival of patients with recurrent nasopharyngeal carcinoma after Gamma Knife radiosurgery according to the use of co-registration with PET-CT and MRI. The difference between the two groups was significant $(p=0.027)$. PET-CT : positron emission tomography-computed tomography, MRI : magnetic resonance imaging. with a low risk of radiation-induced complications. Furthermore, GKS using co-registration with PET-CT and MRI improved local control in patients with recurrent NPC.

Recurrent NPCs are currently treated with FSRT, IMRT, brachytherapy, SRS, and combinations thereof ${ }^{11)}$. FSRT is considered the first-line option for patients with recurrent NPC with previous radiotherapy, but is often associated with radiation-related complications. Although modern radiotherapy techniques have advanced greatly, re-irradiation for recurrent NPC is often difficult because exposing critical structures such as cranial nerves, brain stem, and optic apparatus to radiation can result in serious complications. Extensive resection or additional chemotherapy has not been shown to be as effective against recurrent NPC as other methods. Because of the radiosensitive nature of NPC, SRS has been recommended for certain patients; high conformity and precision SRS with Gamma Knife ${ }^{\circledR}$ (Elekta Inc) was shown to improve tumor control and reduce radiation-related risks.

\section{SRS for recurrent NPC}

NPC carries a poor prognosis and exhibits high recurrence rates that range from $17 \%$ to $23 \%$ depending on the initial tumor status; the 5-year overall survival rate post-salvage treatment for recurrent NPC is $9-30 \%{ }^{9)}$. Conventional radiotherapy is of limited use given the desire to avoid normal organ-atrisk. Boost radiation has become a standard salvage treatment option for residual NPC, while FSRT and SRS are effective salvage treatment modalities as well. Several studies of FSRT and SRS after primary radiotherapy for residual NPC have been performed ${ }^{3,5,8,9,14)}$. FSRT is a modification of SRS that enables fractionated irradiation while preserving the accuracy and dose conformity of SRS. Liu et al. ${ }^{9)}$ reported excellent lo-

Table 3. Summary of previous reports of recurrent nasopharyngeal carcinoma treated with stereotactic radiosurgery

\begin{tabular}{|c|c|c|c|c|c|c|c|}
\hline Study & $\begin{array}{c}\text { No. of } \\
\text { Patients }\end{array}$ & Treatment & $\begin{array}{c}\text { Median margin } \\
\text { dose (Gy) }\end{array}$ & $\begin{array}{l}\text { Local failure-free } \\
\text { survival rate }\end{array}$ & $\begin{array}{l}\text { Overall survival } \\
\text { rate }\end{array}$ & $\begin{array}{c}\text { Toxicity } \\
\text { related SRS }\end{array}$ & $\begin{array}{l}\text { Follow-up } \\
\text { (months) }\end{array}$ \\
\hline Le et al. ${ }^{8)}(2003)$ & 45 & LINAC & 12 & 3-year, $71 \%$ & 3 -year, $75 \%$ & 0 & 35 \\
\hline Chua et al. ${ }^{3)}$ (2006) & 48 & LINAC & 12.5 & 5-year, 47.2\% & 5-year, 46.9\% & $27 \%$ & 54 \\
\hline Chua et al." (2007) & 37 & LINAC & 12.5 & 3-year, 68.3\% & 3-year, $77.5 \%$ & $21.6 \%$ & 42 \\
\hline O'Donnell et al. ${ }^{10)}(2008)$ & 2 & GKS & 14 & 2-year, 100\% & 2-year, 100\% & 0 & 24 \\
\hline Díaz-Martínez et al..6) (2018) & 9 & GKS & 13 & $70 \%$ & Died, 1 patient & $11 \%$ & 13 \\
\hline Present series & 10 & GKS & 18 & 3 -year, 32\% & 3 -year, 77\% & 0 & 37 \\
\hline
\end{tabular}

SRS : stereotactic radiosurgery, LINAC : linear accelerator, GKS : Gamma Knife radiosurgery 
cal control for 136 patients treated with FSRT for residual NPCs after primary radiotherapy; their 5-year disease-free and overall survival rates were $73.6 \%$ and $76.2 \%$, respectively with no severe acute toxicities secondary to FSRT. Xiao et al. ${ }^{14)}$ reported that eight of their 50 patients died of fatal nasopharyngeal hemorrhage after FSRT for recurrent or residual NPCs, whereupon they reduced the fractionated dose; their findings suggested that a high cumulative dose of FSRT and EBRT, previous nasopharyngeal brachytherapy, and nasopharyngeal necrosis elevated the risk of fatal hemorrhage.

SRS was recently introduced as an important additional or alternative salvage treatment option for selected patients with recurrent NPCs. In particular, NPCs that tend to continue recurring require repetitive treatments. GKS can deliver a high dose of radiation while better sparing the normal tissue owing its rapid dose fall-off and is therefore recommended for repetitive salvage therapy. In study of 48 residual NPCs treated by SRS using linear accelerator, Chua et al. ${ }^{5}$ reported 5-year local failure-free survival and overall survival rates of $47.2 \%$ and $46.9 \%$, respectively (Table 3). It was also proposed that patients with good prognostic scores should be treated with SRS given its excellent results ${ }^{3}$. In our study, the 3-year local failure-free and overall survival rates post-GKS were $32 \%$ and $77 \%$, respectively. Although the number of patients in our study was small and the follow-up period was short, our results are consistent with previous FSR or SRS studies.

Studies of treating NPCs with GKS are rare ${ }^{6,10)}$. Díaz-Martínez et al. ${ }^{6}$ found that planned GKS after IMRT in nine patients with NPCs provided excellent local control without acute radiation related toxicity and that good local control rates were achieved in all patients. O'Donnell et al. ${ }^{10)}$ reported that two patients with early diagnosed, locally recurrent NPCs were successfully treated with GKS. The patients remained well without evidence of recurrence or complications 2 years after GKS. Sultanem et al. ${ }^{12}$ found that IMRT showed improved tumor target coverage over conventional radiotherapy with significantly better sparing of the nearby normal structures. The incidences of severe and life-threatening toxicity due to conventional CCRT were $21-55 \%$ in an intergroup trial for $\mathrm{NPC}^{12)}$. IMRT achieved better local control with decreased toxicity; however, studies of IMRT for treating recurrent NPCs are scarce. In a preliminary study, Chua et al. ${ }^{4)}$ reported 1-year locoregional progression-free and overall survival rates of $56 \%$ and $63 \%$, respectively, after re-irradiation with IMRT for locally recurrent NPCs. Late toxicities were common, although the incidences of severe toxicities were relatively low. In our study, there were no symptomatic radiation-related complications caused by salvage GKS. Le et al ${ }^{8}{ }^{8}$ reported that the incidence of late toxicity due to SRS boost after EBRT was low with acceptable levels of severity. Brachytherapy spares the critical organs, increases the radiation dose to the tumor, and improves tumor control in early-state NPC; however, it cannot be used for irradiating the intracranial area or deep parapharyngeal space ${ }^{1)}$. Teo et al. ${ }^{13)}$ reported that brachytherapy was associated with significantly worse local control and survival, although a higher radiation dose via external radiotherapy improved such outcomes.

\section{GKS using co-registration with PET-CT and MRI}

To achieve better tumor control with GKS, it is important to accurately delineate the tumor volume. However, post-irradiation changes on MRI such as edema, fibrosis or loss of tissue plans can be confused with recurrent disease ${ }^{2}$. To prevent this, innovative GKS-related techniques such as co-registration with PET-CT and MRI ought to be considered. In our study, no patient or tumor-related factors, including tumor stage and volume, were significantly associated with the NPC local failure rate of NPC post-GKS. However, outcomes significantly improved using co-registration with PET-CT and MRI when planning GKS. PET-CT is an important modality for radiotherapy planning as well as defining the tumor volume to achieve better tumor control ${ }^{7}$. On GKS planning, we found that co-registered PET-CT more precisely identifies NPC with deep soft tissue. Moreover, when we compared PET-CT with MRI-based planning, we found that the MRIbased target was often not included in the area delineated by the PET-CT-based target. Insufficient coverage of the gross tumor volume results in tumor control failure, especially in NPCs with highly recurrent and infiltrative natures. Irradiation of potential subclinical areas as detected by PET-CT is important for achieving better tumor control. We found that GKS using co-registration with PET-CT and MRI had better tumor control than GKS with conventional MRI-based planning. Hence, we recommend using a PET-CT/MRI fusion image when planning GKS for patients with NPC.

There are several advantages associated with this co-registration technique. One advantage is that co-registered PETCT images provide clear tumor margins than MRI or CT 
alone. MRI is necessary for delineating tumor margin in GKS planning, and is superior to CT; however, MRI contrast enhancement often complicates distinguishing between a tumor, inflammation, and radiation-induced fibrosis. Second, the treatment plan can be adjusted so that the maximum radiation dose is applied to the "hot spot" identified on the PETCT scan. Third, PET-CT for treatment plan is useful for decision making, when the same modality will be used for followup, especially in terms of identifying tumor recurrence and distinguishing between malignant tissue and radiation necrosis. On the other hand, the majority of PET imaging centers is not equipped to acquire frame-based stereotactic PET-CT; therefore, we used co-registration with PET-CT and MRI for GKS instead of frame-based stereotactic PET-CT. However, additional efforts are needed to reduce inaccuracies in co-registered images. Furthermore, PET-CT is often too expensive to utilize solely for use in GKS planning, and may be more expensive than the GKS itself. However, in the majority of patients with NPC, PET-CT is already performed for tumor staging and determining the extent of invasion before treatment, hence PET-CT images acquired at recurrence can be used for co-registration.

\section{Study limitations}

This study is one of very few to date to investigate salvage GKS for recurrent NPC. However, our analyses were limited by the small amount of data as well as the retrospective, single-center nature of the study. A multicenter study should be performed to acquire sufficient data on GKS for recurrent NPCs to better assess the efficacy of GKS. Further investigations of new techniques are also acquired to improve salvage GKS treatment outcomes.

\section{CONCLUSION}

GKS is an acceptable salvage treatment option for patients with recurrent NPC who were previously treated with radiation therapy. Our results suggest that GKS using co-registration with PET-CT and MRI for dose planning may be helpful for improving NPC local control despite this disease's poor prognosis.

\section{CONFLICTS OF INTEREST}

No potential conflict of interest relevant to this article was reported.

\section{INFORMED CONSENT}

Informed consent was obtained from all individual participants included in this study.

\section{AUTHOR CONTRIBUTIONS}

\author{
Conceptualization : CL, SHP \\ Data curation : SYY, KSP \\ Formal analysis : SHP \\ Funding acquisition : SHP \\ Methodology: JHH, SKH \\ Project administration : SHP \\ Visualization: JHH, SKH \\ Writing - original draft : CL \\ Writing - review \& editing : SHP
}

\section{ORCID}

Chaejin Lee https://orcid.org/0000-0002-4089-987X

Seong-Hyun Park https://orcid.org/0000-0002-0982-5338

Sang-Youl Yoon https://orcid.org/0000-0003-2207-3481

Ki-Su Park https://orcid.org/0000-0002-4829-6299

Jeong-Hyun Hwang https://orcid.org/0000-0002-5306-6922

Sung-Kyoo Hwang https://orcid.org/0000-0003-0146-5232

\section{References}

1. Chang JT, See LC, Tang SG, Lee SP, Wang CC, Hong JH : The role of brachytherapy in early-stage nasopharyngeal carcinoma. Int J Radiat Oncol Biol Phys 36 : 1019-1024, 1996

2. Chong VF, Fan YF : Detection of recurrent nasopharyngeal carcinoma: MR imaging versus CT. Radiology 202 : 463-470, 1997

3. Chua DT, Sham JS, Hung KN, Leung LH, Au GK : Predictive factors of tumor control and survival after radiosurgery for local failures of nasopharyngeal carcinoma. Int J Radiat Oncol Biol Phys 66 : 1415-1421, 
2006

4. Chua DT, Sham JS, Leung LH, Au GK : Re-irradiation of nasopharyngeal carcinoma with intensity-modulated radiotherapy. Radiother Oncol 77 : 290-294, 2005

5. Chua DT, Wei WI, Sham JS, Hung KN, Au GK : Stereotactic radiosurgery versus gold grain implantation in salvaging local failures of nasopharyngeal carcinoma. Int J Radiat Oncol Biol Phys 69 : 469-474, 2007

6. Díaz-Martínez JA, Esquenazi Y, Martir M, Citardi MJ, Karni RJ, Blanco $\mathrm{Al}$ : Planned gamma knife boost after chemoradiotherapy for selected sinonasal and nasopharyngeal cancers. World Neurosurg 119 : e467e474, 2018

7. King AD, Ma BB, Yau YY, Zee B, Leung SF, Wong JK, et al. : The impact of $18 \mathrm{~F}-\mathrm{FDG}$ PET/CT on assessment of nasopharyngeal carcinoma at diagnosis. Br J Radiol 81 : 291-298, 2008

8. Le QT, Tate D, Koong A, Gibbs IC, Chang SD, Adler JR, et al. : Improved local control with stereotactic radiosurgical boost in patients with nasopharyngeal carcinoma. Int J Radiat Oncol Biol Phys 56 : 1046-1054, 2003

9. Liu F, Xiao JP, Xu GZ, Gao L, Xu YJ, Zhang Y, et al. : Fractionated stereotactic radiotherapy for 136 patients with locally residual nasopharyngeal carcinoma. Radiat Oncol 8 : 157, 2013

10. O'Donnell HE, Plowman PN, Khaira MK, Alusi G : PET scanning and Gamma Knife radiosurgery in the early diagnosis and salvage "cure" of locally recurrent nasopharyngeal carcinoma. Br J Radiol 81 : e26-e30, 2008

11. Smee RI, Meagher NS, Broadley K, Ho T, Williams JR, Bridger GP : Recurrent nasopharyngeal carcinoma: current management approaches. Am J Clin Oncol 33 : 469-473, 2010

12. Sultanem K, Shu HK, Xia P, Akazawa C, Quivey JM, Verhey LJ, et al. : Three-dimensional intensity-modulated radiotherapy in the treatment of nasopharyngeal carcinoma: the University of California-San Francisco experience. Int J Radiat Oncol Biol Phys 48 : 711-722, 2000

13. Teo PM, Kwan WH, Yu P, Lee WY, Leung SF, Choi P : A retrospective study of the role of intracavitary brachytherapy and prognostic factors determining local tumour control after primary radical radiotherapy in 903 non-disseminated nasopharyngeal carcinoma patients. Clin Oncol (R Coll Radiol) 8 : 160-166, 1996

14. Xiao J, Xu G, Miao Y : Fractionated stereotactic radiosurgery for 50 patients with recurrent or residual nasopharyngeal carcinoma. Int J Radiat Oncol Biol Phys 51 : 164-170, 2001 